\title{
Proof of the ionization conjecture in a reduced Hartree-Fock model
}

\author{
Jan Philip Solovej * \\ Department of Mathematics, University of Michigan, Ann Arbor, MI 48109, USA
}

Oblatum 3-V-1990

Summary. The ionization conjecture for atomic models states that the ionization energy and maximal excess charge are bounded by constants independent of the nuclear charge. We prove this for the Hartree-Fock model without the exchange term.

\section{Introduction}

One of the most challenging problems in mathematical physics is to try to understand the experimental fact that atoms can only carry a very small net negative charge. It seems that a neutral atom can only bind one or two extra electrons.

Over the last decade the research into this problem has been extensive, see [BL], [FS 1], [L 3], [LSST], [R 1-2], [SSS], [Si 1-3]. The best known result is due to Lieb [L 3], it says that the total number $N$ of electrons that an atom with nuclear charge $Z$ can bind satisfies the bound

$$
N<2 Z+1
$$

For $Z=1$ this gives the correct bound. In the asymptotic limit $Z \rightarrow \infty$, (1) can be improved ([FS1], [SSS]) to the following bound on the excess charge $Q=N-Z$

$$
Q \leqq \text { const } Z^{1-\varepsilon},
$$

for some $\varepsilon$ with $0<\varepsilon \leqq 2 / 3$ (that one can choose $\varepsilon=2 / 3$ has recently been announced in [FS 2], this also follows from the method in [SSS], if one compares with the model presented here, and use the main results below). However, none of these results come close to explaining $Q \leqq 1$ or 2 . The lack of understanding is so great that to the best of my knowledge not even a heuristic argument for $Q \leqq C$, with $C$ independent of $Z$ has been given.

* Work supported in part by the U.S. National Science Foundation grant DMS-9002416 Present address: Department of Mathematics, University of Toronto, Toronto M5S 1A1, Cana$\mathrm{da}$ 
In this paper we prove this fact in an atomic model whose complexity is so great that it mimics the true quantum theory to a very high degree. The constant bound on $Q$ has previously been established in only a few much simpler models, the Thomas-Fermi model [LS], the Thomas-Fermi-von Weizsäcker model [BL], and the Hellmann model [SW].

The complexity of the model studied here and the nature of the proof given is such that I feel it might very well indicate the right approach to the full problem.

The model we study is given by an energy functional defined on what is called admissible density matrices. An admissible density matrix $\gamma$ is a trace class operator

$$
\gamma: L^{2}\left(\mathbb{R}^{3} ; \mathbb{C}^{2}\right) \rightarrow L^{2}\left(\mathbb{R}^{3} ; \mathbb{C}^{2}\right)
$$

satisfying the operator inequality

$$
0 \leqq \gamma \leqq \mathbf{1}
$$

Such an operator can be written as $\gamma=\sum_{k} \lambda_{k} \varphi_{k} \otimes \bar{\varphi}_{k}$, where $\left\{\varphi_{k}\right\}$ is an orthonormal family in $L^{2}\left(\mathbb{R}^{3} ; \mathbb{C}^{2}\right)$ and $0 \leqq \lambda_{k} \leqq 1$. We can then define the density

$$
\rho_{\gamma}(x)=\sum_{k} \lambda_{k}\left|\varphi_{k}(x)\right|^{2} \in L^{1}\left(\mathbb{R}^{3}\right) .
$$

The Reduced-Hartree-Fock functional is

$$
\mathscr{E}_{\mathrm{RHF}}(\gamma)=\operatorname{Tr}\left[h_{\mathrm{z}} \gamma\right]+D\left(\rho_{\gamma}, \rho_{\gamma}\right),
$$

where $h_{Z}=-\Delta-Z /|x|$ and

$$
D(f, g)=\frac{1}{2} \int f(x)|x-y|^{-1} g(y) d x d y, \quad f, g \in L^{1}\left(\mathbb{R}^{3}\right) .
$$

The real Hartree-Fock model which is so widely used in physics and chemistry is given by the functional

$$
\mathscr{E}_{\mathrm{HF}}(\gamma)=\mathscr{E}_{\mathrm{RHF}}(\gamma)-\frac{1}{2} \int|\gamma(x, y)|^{2}|x-y|^{-1} d x d y .
$$

Here $\gamma(x, y)$ is the integral kernel representing $\gamma$. The last term in (8) is called the exchange term.

Usually the Hartree-Fock functional is only defined on projection operators. However, it was proved in [L2] that minimizing $\mathscr{E}_{\mathrm{HF}}$ over admissible density matrices gives the same result as minimizing over projections only. This fact seems not to hold in general for $\mathscr{E}_{\mathrm{RHF}}$, see also Corollary 2 below. The reduced Hartree-Fock model is somewhat similar to the model originally introduced by Hartree in $[\mathrm{H}]$.

The energy of an atom with $N$ electrons ( $N$ not necessarily an integer) and nuclear charge $Z$ in the reduced Hartree-Fock model is

$$
E_{\mathrm{RHF}}(N, Z)=\inf \left\{\mathscr{E}_{\mathrm{RHF}}(\gamma) \mid \gamma \in G, \operatorname{Tr} \gamma \leqq N\right\},
$$


where

$$
G=\left\{\gamma \text { admissible } \mid \operatorname{Tr}(-\Delta \gamma)<\infty, D\left(\rho_{\gamma}, \rho_{p}\right)<\infty\right\}
$$

In appendix A we study the minimization problem of (9). The result is summarized in

Theorem 1 (a) There exists $N_{c}(Z)$ with $Z \leqq N_{c}(Z) \leqq 2 Z$ such that for all $0<N$ $\leqq N_{c}(Z), E_{\mathrm{RHF}}(N, Z)=\mathscr{E}_{\mathrm{RHF}}(\gamma)$ for some $\gamma \in G$ with $\mathrm{Tr}_{\mathrm{r}} \mathrm{y}=\mathrm{N}$.

(b) $N \mapsto E_{\mathrm{RHF}}(N, Z)$ is convex, non-increasing and constant for $N \geqq N_{c}(Z)$.

(c) The density $\rho_{y}$ is uniquely determined and is spherically symmetric.

(d) $\gamma$ can be written as

$$
\gamma=\sum_{k=1}^{\infty} \lambda_{k} \varphi_{k} \otimes \bar{\varphi}_{k}, \quad 0 \leqq \lambda_{k} \leqq 1
$$

where each $\varphi_{k}$ with $\lambda_{k} \neq 0$ is a normalized eigenfunction of

$$
h_{Z}^{\gamma}=-\Delta-\frac{Z}{|x|}+\rho_{\gamma} *|x|^{-1},
$$

(* denotes convolution).

(e) The energy $E_{\mathrm{RHF}}$ approximates the true quantum energy $E_{Q}$;

$$
\left(1+C_{\lambda} Z^{-2 / 3}\right) E_{\mathrm{RHF}}(N, Z) \leqq E_{Q}(N, Z) \leqq E_{\mathrm{RHF}}(N, Z)
$$

if $N=\lambda Z$ for some constant $0<\lambda \leqq 1$.

Except for a few differences the corresponding theorem for Hartree-Fock was proved in [LS 2]. From this theorem we easily conclude

Corollary 2. If $\gamma=\sum \lambda_{k} \varphi_{k} \otimes \bar{\varphi}_{k}$ is an absolute minimizer then $\lambda_{k}=1$ or 0 unless $h_{Z}^{\gamma} \varphi_{k}=0$.

Proof. We know that $\left(\varphi_{j}, h_{Z}^{\gamma} \varphi_{j}\right) \leqq 0$. If $\left(\varphi_{j}, h_{Z}^{\gamma} \varphi_{j}\right)<0$ and $0<\lambda_{j}<1$ we get

$$
\frac{\partial}{\partial \lambda_{j}} \mathscr{E}_{\mathrm{RHF}}(\gamma)=\left(\varphi_{j}, h_{Z}^{y} \varphi_{j}\right)<0,
$$

hence $\gamma$ cannot be the absolute minimizer.

As stated earlier we will prove a constant bound on the excess charge

$$
Q_{c}(Z)=N_{c}(Z)-Z
$$

But we will in fact conclude this bound from a much stronger result which we will now describe.

In the rest of this paper we will let $\gamma$ denote an absolute minimizer. Define $v(R)$, the screened nuclear charge at radius $R$ and the potential $\varphi_{\mathrm{RHF}}$ by

$$
v(R)=Z-\int_{|x| \leqq R} \rho_{\gamma}(x) d x
$$


and

$$
\varphi_{\mathrm{RHF}}(x)=\frac{Z}{|x|}-\rho_{\gamma} *|x|^{-1}
$$

Our main result is

Theorem 3. For all $\delta>0$ there exist $\alpha, D>0$ such that for all $R$ satisfying

$$
\alpha Z^{-1 / 3} \leqq R \leqq D
$$

we have

$$
\left(324 \pi^{2}-\delta\right) R^{-3} \leqq v(R) \leqq\left(324 \pi^{2}+\delta\right) R^{-3},
$$

and for all $x$ such that $R=|x|$ satisfies (17)

$$
\left(81 \pi^{2}-\delta\right)|x|^{-4} \leqq \varphi_{\mathrm{RHF}}(x) \leqq\left(81 \pi^{2}+\delta\right)|x|^{-4} .
$$

The constants $324 \pi^{2}$ and $81 \pi^{2}$ come from the Thomas-Fermi (TF) theory. It is in fact easy to prove the above Theorem in the TF theory (see Theorem B 3).

The result corresponding to (19) in the Thomas-Fermi-von Weizsäcker theory was proved in [So], by a method completely different from what will be presented here. In [So] (19) was the key to proving universality, i.e., the existence of a limiting electron density for large atoms.

The idea to prove Theorem 3 is to use a renormalization scheme comparing with TF type models on different length scales. By comparison with the regular TF model it is indeed, as we will see, easy to show that (18) and (19) hold for $\alpha Z^{-\frac{1}{3}} \leqq R \leqq \beta Z^{-\frac{1}{3}(1-\varepsilon)}$, for some $\varepsilon>0$. Using this we compare the density outside $\{|x| \leqq R\}$ with the density of what we call an exterior TF model. This will give (18) and (19) for $R$ in an interval of larger scale. This scheme will converge to give Theorem 3. In Appendix B we define and study exterior TF models.

From Theorem 3 it will be easy to conclude

Theorem 4. For all $Z$ we get the following bounds on the excess charge $Q_{c}(Z)$, and ionization energy $I(Z)=E_{\mathrm{RHF}}\left(N_{c}(Z)-1, Z\right)-E_{\mathrm{RHF}}\left(N_{c}(Z), Z\right) \geqq 0$

$$
Q_{c}(Z) \leqq C_{Q} \text { and } I(Z) \leqq C_{I},
$$

where $C_{Q}$ and $C_{I}$ are independent of $Z$.

The paper is organized as follows: In Sections 2 and 3 we compare with the exterior TF model. Section 2 gives an upper bound to $E_{\mathrm{RHF}}$ and Sect. 3 a lower bound. In Sect. 4 we prove Theorem 3 and in Sect. 5, Theorem 4.

\section{Comparison with exterior TF density: upper bound}

Let $\gamma$ be an absolute minimizer of $\mathscr{E}_{\mathrm{RHF}}$. We will define a trial density matrix by changing $\gamma$ in an exterior region. We use the method of coherent states (see [L 1], [L 4] and [T]). 
For all $R_{1}>0$ and $0<r<\frac{1}{4} R_{1}$ choose two spherically symmetric $C^{\infty}$-functions $\theta_{ \pm}: \mathbb{R}^{3} \rightarrow \mathbb{R}_{+} \cup\{0\}$ such that $\theta_{+}^{2}+\theta_{-}^{2}=1$ and satisfying

$$
\theta_{+}(x)=0 \quad \text { if }|x| \leqq R_{1}-r \quad \text { and } \quad \theta_{+}(x)=1 \quad \text { if }|x| \geqq R_{1}+r
$$

with

$$
\left|\nabla \theta_{ \pm}\right| \leqq C_{\theta} r^{-1} .
$$

Define a trial density matrix by

$$
\tilde{\gamma}\left(x, x^{\prime}\right)=\theta_{-}(x) \gamma\left(x, x^{\prime}\right) \theta_{-}\left(x^{\prime}\right)+\theta_{+}(x) K\left(x, x^{\prime}\right) \theta_{+}\left(x^{\prime}\right)
$$

where

$$
K\left(x, x^{\prime}\right)=I_{\sigma}(2 \pi)^{-3} \int d p d q g(x-q) \overline{g\left(x^{\prime}-q\right)} M(p, q) e^{i p\left(x-x^{\prime}\right)} .
$$

Here $I_{\sigma}$ is the identity on $\mathbb{C}^{2}$ and

$$
g(x)=r^{-3 / 2} g_{1}(x / r) \quad g_{1}=\left\{\begin{array}{cc}
(2 \pi)^{-1 / 2}|x|^{-1} \sin (\pi|x|), & |x| \leqq 1 \\
0, & |x|>1
\end{array}\right.
$$

then $\int g(x)^{2} d x=1$.

The function $M$ is defined by

$$
M(p, q)=\theta\left(\left(3 \pi^{2}\right)^{2 / 3} \rho_{u}(q)^{2 / 3}-p^{2}\right),
$$

where $\theta$ is the function

$$
\theta(t)=\left\{\begin{array}{ll}
1 & \text { if } t \geqq 0 \\
0 & \text { if } t<0
\end{array} .\right.
$$

$\rho_{u}$ is a non-negative function in $L^{1}\left(\mathbb{R}^{3}\right) \cap L^{5 / 3}\left(\mathbb{R}^{3}\right)$, to be chosen later, and satisfying

$$
\operatorname{supp} \rho_{u} \subseteq\left\{x|| x \mid \geqq R_{1}+2 r\right\}
$$

We have to check the admissibility of $\tilde{\gamma}$. Given $f \in L^{2}\left(\mathbb{R}^{3} ; \mathbb{C}^{2}\right)$ then with (, ) denoting inner product

$$
(f, \tilde{\gamma} f)=\left(f, \theta_{-} \gamma \theta_{-} f\right)+\left(f, \theta_{+} K \theta_{+} f\right) .
$$

Since $\gamma$ is admissible $0 \leqq\left(f, \theta_{-} \gamma \theta_{-} f\right) \leqq\left\|\theta_{-} f\right\|^{2}$.

A straightforward computation using Parseval's identity gives

$$
0 \leqq\left(f, \theta_{+} K \theta_{+} f\right) \leqq\left\|\theta_{+} f\right\|^{2} .
$$

Thus $0 \leqq(f, \tilde{\gamma} f) \leqq\|f\|^{2}$, i.e.,

$$
0 \leqq \tilde{\gamma} \leqq 1
$$

The density corresponding to $K$ is

$$
\rho_{K}(x)=K(x, x)=\rho_{u} *|g|^{2} .
$$


It is then clear that $\tilde{\gamma}$ is trace class and

$$
\begin{aligned}
\rho_{\tilde{y}}(x) & =\theta_{-}(x)^{2} \rho_{\gamma}(x)+\theta_{+}(x)^{2} \rho_{u} *|g|^{2} \\
& =\theta_{-}(x)^{2} \rho_{\gamma}(x)+\rho_{u} *|g|^{2}
\end{aligned}
$$

where we have used the support properties (1) and (8). Indeed $\theta_{+} K \theta_{+}=K$.

We will now compute $\mathscr{E}_{\mathrm{RHF}}(\tilde{\gamma})$,

$$
\operatorname{Tr}[-\Delta \tilde{\gamma}]=\operatorname{Tr}\left[-\Delta\left(\theta_{-} \gamma \theta_{-}\right)\right]+\operatorname{Tr}[-\Delta K] .
$$

An easy computation gives

$$
\begin{aligned}
\operatorname{Tr}[-\Delta K] & =\int|\nabla g|^{2} d x \int \rho_{u}(x) d x+\frac{3}{5}\left(3 \pi^{2}\right)^{2 / 3} \int \rho_{u}(x)^{5 / 3} d x \\
& =\pi r^{-2} \int \rho_{u}(x) d x+\frac{3}{5}\left(3 \pi^{2}\right)^{2 / 3} \int \rho_{u}(x)^{5 / 3} d x .
\end{aligned}
$$

We also get with $V=Z|x|^{-1}$

$$
\begin{aligned}
\operatorname{Tr}[V K] & =\int \rho_{K}(x) V(x) d x=\int \rho_{u} *|g|^{2} \cdot \frac{Z}{|x|} d x \\
& =Z \int|x|^{-1} \rho_{u}(u) d x
\end{aligned}
$$

since supp $g \cap \operatorname{supp} \rho_{u}=\emptyset$ and $g$ is spherically symmetric with $\int|g|^{2}=1$.

Thus

$$
\begin{aligned}
\operatorname{Tr}\left[h_{Z} \tilde{\gamma}\right]= & \operatorname{Tr}\left[h_{Z}\left(\theta_{-} \gamma \theta_{-}\right)\right]+\frac{3}{5}\left(3 \pi^{2}\right)^{2 / 3} \int \rho_{u}(x)^{5 / 3} d x \\
& -Z \int|x|^{-1} \rho_{u}(x) d x+\pi r^{-2} \int \rho_{u}(x) d x .
\end{aligned}
$$

For the last term in $\mathscr{E}_{\mathrm{RHF}}$ we find

$$
\begin{aligned}
D\left(\rho_{\hat{\gamma}}, \rho_{\hat{\gamma}}\right)=D\left(\theta_{-}^{2} \rho_{y}, \theta_{-}^{2} \rho_{y}\right) & +2 D\left(\theta_{-}^{2} \rho_{\gamma}, \rho_{u} *|g|^{2}\right) \\
& +D\left(\rho_{u} *|g|^{2}, \rho_{u} *|g|^{2}\right) .
\end{aligned}
$$

Now $D\left(\rho_{u} *|g|^{2}, \rho_{u} *|g|^{2}\right) \leqq D\left(\rho_{u}, \rho_{u}\right)$. Since $\theta_{-}^{2} \rho_{\gamma}$ is spherically symmetric

$$
2 D\left(\theta_{-}^{2} \rho_{\gamma}, \rho_{u} *|g|^{2}\right)=(Z-\bar{v}) \int|x|^{-1} \rho_{u}(x) d x
$$

where

$$
\bar{v}=Z-\int \theta_{-}^{2} \rho_{y}(x) d x
$$

We have

$$
E_{\mathrm{RHF}}^{\min } \equiv \mathscr{E}_{\mathrm{RHF}}(\gamma) \leqq \mathscr{E}_{\mathrm{RHF}}(\tilde{\gamma}) \leqq \mathscr{E}_{\mathrm{RHF}}\left(\theta_{-} \gamma \theta_{-}\right)+\mathscr{E}_{\mathrm{TF}}\left(\rho_{u}\right)+\pi r^{-2} \int \rho_{u}(x) d x,
$$
where $\mathscr{E}_{\mathrm{TF}}$ is defined in Appendix B. 


\section{Comparison with exterior TF density: lower bound}

As before let $\gamma$ be an absolute minimizer, i.e.,

$$
\begin{aligned}
E_{\mathrm{RHF}}^{\min } & =\mathscr{E}_{\mathrm{RHF}}(\gamma)=\operatorname{Tr}\left[h_{z} \gamma\right]+D\left(\rho_{\gamma}, \rho_{\gamma}\right) \\
& =\operatorname{Tr}\left[\left(\theta_{-}^{2}+\theta_{+}^{2}\right) h_{Z} \gamma\right]+D\left(\rho_{\gamma}, \rho_{\gamma}\right) .
\end{aligned}
$$

It is easy to prove the following version of the IMS-formula

$$
\operatorname{Tr}\left[\left(\theta_{-}^{2}+\theta_{+}^{2}\right)(-\Delta \gamma)\right]=\operatorname{Tr}\left[-\Delta\left(\theta_{-} \gamma \theta_{-}\right)-\Delta\left(\theta_{+} \gamma \theta_{+}\right)\right]-\operatorname{Tr}\left[\left(\left(\nabla \theta_{-}\right)^{2}+\left(\nabla \theta_{+}\right)^{2}\right) \gamma\right] .
$$

Thus

$$
\begin{aligned}
E_{\mathrm{RHF}}^{\min }= & \operatorname{Tr}\left[h_{Z}\left(\theta_{-} \gamma \theta_{-}\right)+h_{z}\left(\theta_{+} \gamma \theta_{+}\right)\right]-\operatorname{Tr}\left[\left(\left(\nabla \theta_{-}\right)^{2}+\left(\nabla \theta_{+}\right)^{2}\right) \gamma\right] \\
& +D\left(\theta_{-}^{2} \rho_{\gamma}, \theta_{-}^{2} \rho_{\gamma}\right)+2 D\left(\theta_{-}^{2} \rho_{\gamma}, \theta_{+}^{2} \rho_{\gamma}\right)+D\left(\theta_{+}^{2} \rho_{\gamma}, \theta_{+}^{2} \rho_{\gamma}\right) \\
= & \mathscr{E}_{\mathrm{RHF}}\left(\theta_{-} \gamma \theta_{-}\right)+\operatorname{Tr}\left[h_{Z}\left(\theta_{+} \gamma \theta_{+}\right)\right]+2 D\left(\theta_{-}^{2} \rho_{\gamma}, \theta_{+}^{2} \rho_{\gamma}\right) \\
& +D\left(\theta_{+}^{2} \rho_{\gamma}, \theta_{+}^{2} \rho_{\gamma}\right)-\operatorname{Tr}\left[\left(\left(\nabla \theta_{-}\right)^{2}+\left(\nabla \theta_{+}\right)^{2}\right) \gamma\right] .
\end{aligned}
$$

We write

$$
\begin{aligned}
2 D\left(\theta_{-}^{2} \rho_{\gamma}, \theta_{+}^{2} \rho_{\gamma}\right) & =\int\left(\theta_{-}^{2} \rho_{\gamma}\right) *|x|^{-1}\left(\theta_{+}^{2} \rho_{\gamma}\right)(x) d x \\
& =(Z-\bar{v}) \int|x|^{-1} \theta_{+}^{2} \rho_{\gamma}(x)+\int\left(\left(\theta_{-}^{2} \rho_{\gamma}\right) *|x|^{-1}-\frac{Z-\bar{v}}{|x|}\right) \theta_{+}^{2} \rho_{\gamma}(x) d x .
\end{aligned}
$$

Since $\theta_{-}^{2} \rho_{\gamma}$ is spherically symmetric we get using (2.15)

$$
\begin{aligned}
& \int\left(\left(\theta_{-}^{2} \rho_{\gamma}\right) *|x|^{-1}-\frac{Z-\bar{v}}{|x|}\right)\left(\theta_{+}^{2} \rho_{\gamma}\right)(x) d x \\
& =\int_{\substack{R_{1}-r \leq|x| \leq R_{1}+r \\
|y| \geqq|x|}} \theta_{-}^{2} \rho_{y}(y)\left[\frac{1}{|y|}-\frac{1}{|x|}\right] \theta_{+}^{2} \rho_{\gamma}(x) d x d y \\
& \geqq-\Delta Q\left(R_{1}, r\right)^{2}\left(\frac{1}{R_{1}-r}-\frac{1}{R_{1}+r}\right),
\end{aligned}
$$

where

$$
\Delta Q\left(R_{1}, r\right)=\int_{R_{1}-r \leqq|x| \leqq R_{1}+r} \rho_{y}(x) d x
$$

We obtain

$$
2 D\left(\theta_{-}^{2} \rho_{\gamma}, \theta_{+}^{2} \rho_{\gamma}\right) \geqq \int \frac{Z-\bar{v}}{|x|} \theta_{+}^{2} \rho_{\gamma} d x-\Delta Q\left(R_{1}, r\right)^{2} \frac{2 r}{\left(R_{1}-r\right)^{2}} .
$$

Hence from (1) and (2.2)

$$
\begin{aligned}
E_{\mathrm{RHF}}^{\min } \geqq \mathscr{E}_{\mathrm{RHF}}\left(\theta_{-} \gamma \theta_{-}\right)+\operatorname{Tr}\left[h_{v}\left(\theta_{+} \gamma \theta_{+}\right)\right]+D\left(\theta_{+}^{2} \rho_{\gamma}, \theta_{+}^{2} \rho_{\gamma}\right) & -C_{\theta} r^{-2} \Delta Q\left(R_{1}, r\right)-4 R_{1}^{-2} r \Delta Q\left(R_{1}, r\right)^{2},
\end{aligned}
$$

we have also used $r<\frac{1}{4} R_{1}$. 
For $\rho_{L} \in L^{1}\left(\mathbb{R}^{3}\right) \cap L^{5 / 3}\left(\mathbb{R}^{3}\right)$ a non-negative function, to be chosen later, we write

$$
D\left(\theta_{+}^{2} \rho_{\gamma}, \theta_{+}^{2} \rho_{\gamma}\right)=2 D\left(\rho_{L}, \theta_{+}^{2} \rho_{\gamma}\right)+D\left(\theta_{+}^{2} \rho_{\gamma}-\rho_{L}, \theta_{+}^{2} \rho_{\gamma}-\rho_{L}\right)-D\left(\rho_{L}, \rho_{L}\right) .
$$

Hence from (4)

(6) $E_{\mathrm{RHF}}^{\min } \geqq \mathscr{E}_{\mathrm{RHF}}\left(\theta_{-} \gamma \theta_{-}\right)+\operatorname{Tr}\left[\bar{h}_{\bar{v}}\left(\theta_{+} \gamma \theta_{+}\right)\right]-D\left(\rho_{L}, \rho_{L}\right)$

$$
+D\left(\theta_{+}^{2} \rho_{\gamma}-\rho_{L}, \theta_{+}^{2} \rho_{\gamma}-\rho_{L}\right)-C_{\theta} r^{-2} \Delta Q\left(R_{1}, r\right)-4 R_{1}^{-2} r \Delta Q\left(R_{1}, r\right)^{2},
$$

where

$$
\bar{h}_{\bar{v}}=-\Delta-\left(\frac{\bar{v}}{|x|}-\rho_{L} *|x|^{-1}\right)
$$

We will now find a lower bound to $\operatorname{Tr}\left[\bar{h}_{\bar{v}}\left(\theta_{+} \gamma \theta_{+}\right)\right] . \theta_{+} \gamma \theta_{+}$is a trace class operator in $L^{2}\left(\mathbb{R}^{3} ; \mathbb{C}^{2}\right)$ with

$$
0 \leqq\left(\theta_{+} \gamma \theta_{+}\right) \leqq 1 \text { and } \operatorname{supp} \rho_{\theta_{+} \gamma \theta_{+}} \subseteq\left\{|x| \geqq R_{1}-r\right\} .
$$

Therefore

$$
\operatorname{Tr}\left[\bar{h}_{\tilde{v}}\left(\theta_{+} \gamma \theta_{+}\right)\right] \geqq \text {sum of all negative eigenvalues of } \bar{h}_{\bar{v}}^{D},
$$

here $h_{\bar{v}}^{D}$ is the Dirichlet realization of $\bar{h}_{\bar{v}}$ on $\left\{|x| \geqq R_{1}-r\right\}$.

Writing

$$
\bar{V}=\frac{\bar{v}}{|x|}-\rho_{L} *|x|^{-1}
$$

we get since $r \leqq R_{1} / 4$ using the same coherent states as for the upper bound that for $|x| \geqq R_{1}-r$

$$
\begin{aligned}
\bar{V} *|g|^{2}-\bar{V}= & \bar{v}\left(|g|^{2} *|x|^{-1}-|x|^{-1}\right) \\
& +\rho_{L} *|x|^{-1}-\rho_{L} *|g|^{2} *|x|^{-1} \\
= & \rho_{L} *|x|^{-1}-\rho_{L} *|g|^{2} *|x|^{-1} \geqq 0
\end{aligned}
$$

since $\rho_{L} *|x|^{-1}$ is superharmonic. Hence

$$
h_{\bar{v}}^{D} \geqq-\Delta_{D}-\bar{V} *|g|^{2} .
$$

We will estimate the sum of all the negative eigenvalues of $-\Delta_{D}-\vec{V} *|g|^{2}$ as an operator on $L^{2}\left(\left\{|x| \geqq R_{1}-r\right\} ; \mathbb{C}^{2}\right)$.

Let $m_{1}, \ldots$ be the normalized eigenfunctions. Define

$$
M(p, q)=\sum_{i=1}\left(m_{i}, \Pi_{p q} m_{i}\right),
$$

where $\Pi_{p q}$ is the projection

$$
\Pi_{p q}\left(x, x^{\prime}\right)=I_{\sigma} f_{p q}(x) \overline{f_{p q}\left(x^{\prime}\right)}
$$


for

$$
f_{p q}(x)=g(x-q) e^{i p x}
$$

Since $m_{i}$ is supported in $\left\{|x| \geqq R_{1}-r\right\}$ we see that $M$ has the following properties

$$
\begin{gathered}
M(p, q)=0 \quad \text { if }|q|<R_{1}-2 r \\
0 \leqq M(p, q) \leqq \operatorname{Tr} \Pi_{p q}=2\left(=\operatorname{dim} \mathbb{C}^{2}=\# \text { of spins }\right) .
\end{gathered}
$$

We will choose $\rho_{L}$ such that the number $\tilde{N}$ of negative eigenvalues of $\hbar_{\bar{v}}^{D}$ is finite, see (23) below. Then

$$
(2 \pi)^{-3} \iint d p d q M(p, q) \leqq \tilde{N}
$$

since $(2 \pi)^{-3} \iint d p d q \Pi_{p q}=1$.

We also have the following identities for any function $m \in L^{2}\left(\mathbb{R}^{3} ; \mathbb{C}^{2}\right)$

$$
\begin{gathered}
\int|\nabla m|^{2} d x=(2 \pi)^{-3} \iint d p d q p^{2}\left(m, \Pi_{p q} m\right) \cdots(m, m) \int|\nabla g|^{2} d x \\
\int|m|^{2} \bar{V} *|g|^{2} d x=(2 \pi)^{-3} \iint d p d q \bar{V}(q)\left(m, \Pi_{p q} m\right) .
\end{gathered}
$$

Hence the sum of the negative eigenvalues of $-\Delta_{D}-\bar{V} *|g|^{2}$ is

$$
\begin{aligned}
& (2 \pi)^{-3} \underset{|q| \geqq R_{1}-2 r}{\iint_{1}} d p d q\left\{p^{2}-\bar{V}(q)\right\} M(p, q) \\
& \quad-(2 \pi)^{-3} \int|\nabla \mathbf{g}|^{2} d x \iint d p d q M(p, q) \\
& \geqq(2 \pi)^{-3} \int_{|q| \geqq R_{1}-2 r} d p d q\left\{p^{2}-\bar{V}(q)\right\} \cdot 2 \theta\left(\bar{V}(q)-p^{2}\right)-\tilde{N} \int|\nabla g|^{2} d x,
\end{aligned}
$$

here we have used (14) and (15).

Now we choose $\rho_{L}$, using the notation of Appendix B

$$
\rho_{L}(x)=\rho_{\mathrm{TF}}\left(x, R_{1}-2 r, \bar{v}\right) .
$$

Then for $|x| \geqq R_{1}-2 r$

$$
\bar{V}(x)=\varphi_{\mathrm{TF}}\left(x, R_{1}-2 r, \bar{v}\right)=\left(3 \pi^{2} \rho_{L}\right)^{2 / 3} \geqq 0
$$

and

$$
\int_{|x| \geqq R_{1}-2 \mathbf{r}} \rho_{L}(x) d x=\bar{v} .
$$


The number of negative eigenvalues $\tilde{N}$ can be estimated from a Theorem of Lieb [L5]

$$
\begin{aligned}
\tilde{N} & \leqq\left.\left.\left(3 \pi^{2}\right)^{-1} L \int_{|x| \geqq R_{1}-r}|\bar{V} *| g\right|^{2}\right|^{3 / 2} d x \\
& \leqq\left(3 \pi^{2}\right)^{-1} L \int_{|x| \geqq R_{1}-2 r}|\bar{V}|^{3 / 2} d x \\
& =L \int_{|x| \geqq R_{1}-2 r} \rho_{L}(x) d x=\bar{v} \cdot L .
\end{aligned}
$$

$L$ is a positive constant.

From (19)-(23) we obtain

(24) $\operatorname{Tr}\left[\bar{h}_{v}\left(\theta_{+} \gamma \theta_{+}\right)\right] \geqq \frac{3}{5}\left(3 \pi^{2}\right)^{2 / 3} \int \rho_{L}(x)^{5 / 3} d x-\int \bar{V}(x) \rho_{L}(x) d x-\bar{v} \cdot L \cdot \pi r^{-2}$.

Going back to (6) and recalling (9) we find

$$
\begin{aligned}
E_{\mathrm{RHF}}^{\min } \geqq & \mathscr{E}_{\mathrm{RHF}}\left(\theta_{-} \gamma \theta_{-}\right)+\mathscr{E}_{\mathrm{TF}}\left(\rho_{L}\right)-\bar{v} \cdot L \cdot \pi r^{-2} \\
& +D\left(\rho_{\gamma} \theta_{+}^{2}-\rho_{L}, \rho_{\gamma} \theta_{+}^{2}-\rho_{L}\right)-C_{\theta} r^{-2} \Delta Q\left(R_{1}, r\right)-4 R_{1}^{-2} r \Delta Q\left(R_{1}, r\right)^{2} .
\end{aligned}
$$

We return now to the upper bound (2.16) and make the explicit choice

$$
\rho_{u}(x)=\left\{\begin{array}{cc}
\rho_{L}(x) & \text { for }|x| \geqq R_{1}+2 r \\
0 & \text { for }|x|<R_{1}+2 r
\end{array} .\right.
$$

Then

$$
\mathscr{E}_{\mathrm{TF}}\left(\rho_{u}\right) \leqq \mathscr{E}_{\mathrm{TF}}\left(\rho_{L}\right)+\int_{R_{1}-2 \boldsymbol{r} \leqq|x| \leqq R_{1}+2 \boldsymbol{r}} \frac{\bar{v}}{|x|} \rho_{L}(x) d x
$$

We know that

$$
3 \pi^{2} \rho_{L}(x)=\bar{V}(x)^{3 / 2} \leqq \bar{v}^{3 / 2}|x|^{-3 / 2}
$$

Hence since $r \leqq \frac{1}{4} \bar{R}$ we can estimate the error term in (27)

$$
\int_{R_{1}-2 r \leqq|x| \leqq R_{1}+2 r} \frac{\bar{v}}{|x|} \rho_{L}(x) d x \leqq \frac{16 \sqrt{2}}{3 \pi} r R_{1}^{-\frac{1}{2}} \bar{v}^{5 / 2} .
$$

Thus combining (2.16) and (25) we obtain

$$
\begin{aligned}
& D\left(\rho_{\gamma} \theta_{+}^{2}-\rho_{L}, \rho_{\gamma} \theta_{+}^{2}-\rho_{L}\right) \leqq(L+1) \pi r^{-2} \bar{v}+C_{\theta} r^{-2} \Delta Q\left(R_{1}, r\right) \\
& \quad+4 R_{1}^{-2} r \Delta Q\left(R_{1}, r\right)^{2}+\frac{16 \sqrt{2}}{3 \pi} r R_{1}^{-\frac{1}{2}} \bar{v}^{5 / 2} .
\end{aligned}
$$


In the case where $r=R_{1}=0$ we easily get by copying the argument in [L4]

$$
D\left(\rho_{\gamma}-\rho_{\mathrm{TF}}, \rho_{\gamma}-\rho_{\mathrm{TF}}\right) \leqq C_{0} Z^{\frac{7}{3}(1-\varepsilon)}
$$

where $\varepsilon=\frac{1}{70}$.

We have

Theorem 5. (a) Given $R_{1}, r>0$ with $r \leqq \frac{1}{4} R_{1}$. Choose $\theta_{ \pm}$corresponding to $R_{1}, r$ as described in (2.1.2). If $\gamma$ is the absolute minimizer and $\rho_{L}(x)=\rho_{\mathrm{TF}}\left(x, R_{1}-2 r, \bar{v}\right)$ then the inequality (28) holds, with $\bar{v}$ and $\Delta Q$ defined in (2.15) and (2) respectively.

(b) In the case where $R_{1}=r=0$ we get the inequality (29).

\section{The renormalization procedure}

In this section we will prove Theorem 3 . We begin with

Lemma 6. For every $\delta>0$ there exist $\beta, \alpha>0$ such that for $\alpha Z^{-\frac{1}{3}} \leqq R \leqq \beta Z^{-\frac{1}{3}(1-\varepsilon)}$ we get (1.18) and (1.19).

Proof. Choose $\chi \in C_{0}^{\infty}\left(\mathbb{R}^{3}\right)$ with $\chi(x)=1$ for $|x| \leqq 1$ and $\chi(x)=0$ for $|x| \geqq 1+\lambda$. Let $\chi_{R}(x)=\chi(x / R)$. Then with denoting Fourier transform

$$
\begin{aligned}
& \left|\int \chi_{R}(x)\left(\rho_{\gamma}(x)-\rho_{\mathrm{TF}}(x)\right) d x\right|=\left|\int \hat{\chi}_{R}(p)\left(\rho_{\gamma}-\rho_{\mathrm{TF}}\right)^{\hat{y}}(p) d p\right| \\
& \quad \leqq C\left(\int\left|\hat{\chi}_{R}(p)\right|^{2}|p|^{2} d p\right)^{1 / 2} D\left(\rho_{\gamma}-\rho_{\mathrm{TF}}, \rho_{\gamma}-\rho_{\mathrm{TF}}\right)^{1 / 2} \\
& \quad \leqq C_{0}\left\|\nabla \chi_{R}\right\|_{2} Z^{\frac{7}{6}(1-\varepsilon)} \leqq C_{\lambda}^{\prime} R^{1 / 2} Z^{\frac{7}{6}(1-\varepsilon)} .
\end{aligned}
$$

Here we have used Theorem 5(b). This inequality was an essential ingredient in [FS1] and [SSS].

For $v(R)$ defined in (1.15) we get

$$
v(R)=Z-\int_{|x| \leqq R} \rho_{y}(x) d x=\int \rho_{\mathrm{TF}}(x)-\int_{|x| \leqq R} \rho_{y}(x) d x .
$$

Using the result corresponding to (B.13) for the regular TF theory we easily see that we can find $\alpha$ such that if $R \geqq \alpha Z^{-1 / 3}$ and $\lambda$ is sufficiently small

$$
\begin{aligned}
& -C_{\lambda} R^{1 / 2} Z^{\frac{7}{6}(1-\varepsilon)}+\left(324 \pi^{2}-\frac{\delta}{2}\right) R^{-3} \leqq v(R) \\
& \leqq C_{\lambda} R^{1 / 2} Z^{\frac{7}{6}(1-\varepsilon)}+\left(324 \pi^{2}+\frac{\delta}{2}\right) R^{-3}
\end{aligned}
$$

Thus if $\alpha Z^{-1 / 3} \leqq R \leqq \beta Z^{-1 / 3(1-\varepsilon)}$ we get

$$
\left(-C_{\lambda} \beta^{7 / 2}+324 \pi^{2}-\frac{\delta}{2}\right) R^{-3} \leqq v(R) \leqq\left(C_{\lambda} \beta^{7 / 2}+324 \pi^{2}+\frac{\delta}{2}\right) R^{-3} .
$$

It is therefore clear that we can choose $\beta$ such that (1.18) holds. 
(1.19) is proved in a similar way except that we replace (1) with

$$
\int\left|\nabla \varphi_{\mathrm{RHF}}-\nabla \varphi_{\mathrm{TF}}\right|^{2} d x=D\left(\rho_{\gamma}-\rho_{\mathrm{TF}}, \rho_{\gamma}-\rho_{\mathrm{TF}}\right),
$$

together with the inequality

$$
\left|\varphi_{\mathrm{RHF}}(x)-\varphi_{\mathrm{TF}}(x)\right| \leqq \frac{1}{2}(\pi|x|)^{-1 / 2}\left(\int_{|y| \geqq|x|}\left|\nabla \varphi_{\mathrm{RHF}}-\nabla \varphi_{\mathrm{TF}}\right|^{2} d y\right)^{1 / 2},
$$

which holds because of spherical symmetry.

The Renormalization procedure is given in

Lemma 7. Given $\delta \leqq 0$. Then there exists $D_{1}(\delta)>0$ such that if (1.18) and (1.19) have been proved for $R$ and $Z$ satisfying

$$
\alpha Z^{-\frac{1}{3}} \leqq R \leqq \beta Z^{-\frac{1}{3}(1-\varepsilon)^{n}} \leqq D_{1}(\delta)
$$

where $\alpha$ and $\beta$ are the same as in Lemma 6 , and $n$ is a positive integer, then (1.18) and (1.19) will hold for $R$ satisfying

$$
\alpha Z^{-\frac{1}{3}} \leqq R \leqq \beta Z^{-\frac{1}{3}(1-\varepsilon)^{n+1}} .
$$

Before proving this lemma we show how to use it in the Proof of Theorem 3. Assume $R$ satisfies (1.17) with $D=\min \left\{\beta, D_{1}(\delta)\right\}$. Then since $\lim _{n \rightarrow \infty} \beta Z^{-\frac{1}{3}(1-\varepsilon)^{n}}=\beta$ we can choose a smallest possible $n \geqq 0$ such that

$$
\alpha Z^{-\frac{1}{3}} \leqq R \leqq \beta Z^{-\frac{1}{3}(1-\varepsilon)^{n+1}}
$$

If $n=0$ we are done by Lemma 6 . If $n \geqq 1$ we know that for all $k \leqq n, \beta Z^{-\frac{1}{3}(1-\varepsilon)^{k}}$ $<R \leqq D_{1}(\delta)$. Then starting with Lemma 6 we prove by induction using Lemma 7 that (1.18) and (1.19) hold for all $R_{1}$ satisfying $\alpha Z^{-\frac{1}{3}} \leqq R_{1} \leqq \beta Z^{-\frac{1}{3}(1-\varepsilon)^{n+1}}$ especially for $R$.

Proof of Lemma 7. We assume that $Z$ satisfies (6), and that (6) implies (1.18) and (1.19). We first notice that $\left(\frac{4}{3} \alpha Z^{-\frac{1}{3}}, \frac{4}{5} \beta Z^{-\frac{1}{3}(1-\varepsilon)^{n}}\right)$ is a non-empty interval if $D_{1}(\delta)<\frac{3}{5} \beta^{1 / \varepsilon} \alpha^{1-1 / \varepsilon}$. Indeed

$$
\frac{4}{5} \beta Z^{-\frac{1}{3}(1-\varepsilon)} \cdot\left[\frac{4}{3} \alpha Z^{-\frac{1}{3}}\right]^{-1}=\frac{3}{5} \beta \alpha^{\varepsilon-1}\left(\alpha Z^{-\frac{1}{3}}\right)^{-\varepsilon} \geqq \frac{3}{5} \beta \alpha^{\varepsilon-1} D_{1}(\delta)^{-\varepsilon}>1 .
$$

Now for any $R_{1} \in\left(\frac{4}{3} \alpha Z^{-\frac{1}{3}}, \frac{4}{5} \beta Z^{-\frac{1}{3}(1-\varepsilon)^{n}}\right)$ define

$$
r=R_{1}^{1+2 / 3} .
$$


Then if $\left(\frac{4}{5} D_{1}(\delta)\right)^{2 / 3} \leqq \frac{1}{4}$ we get $r \leqq \frac{1}{4} R_{1}$.

We choose $\theta_{ \pm}$for $R_{1}, r$ as described in Sect. 2 . Then since $R_{1}-r$ and $R_{1}+r$ satisfy

(6) we can use (1.18) to get the following estimate for $\bar{v}=Z-\int \theta_{-}(x)^{2} \rho_{\gamma}(x) d x$

$$
\left(324 \pi^{2}-\delta\right)\left(\frac{5}{4}\right)^{-3} R_{1}^{-3} \leqq \bar{v} \leqq\left(324 \pi^{2}+\delta\right)\left(\frac{3}{4}\right)^{-3} R_{1}^{-3} .
$$

Furthermore from (3.2)

$$
\begin{aligned}
\Delta Q\left(R_{1}-r\right) & \leqq v\left(R_{1}-r\right)-v\left(R_{1}+r\right) \\
& \leqq\left[\left(324 \pi^{2}+\delta\right)\left(\frac{3}{4}\right)^{-3}-\left(324 \pi^{2}-\delta\right)\left(\frac{5}{4}\right)^{-3}\right] R_{1}^{-3}=c_{\delta}^{\prime} R_{1}^{-3} .
\end{aligned}
$$

Notice especially that

$$
\left(R_{1}-2 r\right)^{3} \bar{v} \geqq \frac{\bar{v} R_{1}^{3}}{8} \geqq \frac{1}{8}\left(\frac{5}{4}\right)^{-3}\left(324 \pi^{2}-\delta\right) .
$$

We get from Theorem 5 using (8)-(10)

$$
D\left(\rho_{\gamma} \theta_{+}^{2}-\rho_{L}, \rho_{\gamma} \theta_{+}^{2}-\rho_{L}\right) \leqq c_{\delta} R_{1}^{-7+\frac{1}{3}}\left(R_{1}^{1 / 3}+R_{1}^{2 / 3}\right) \leqq C_{\delta} D_{1}(\delta)^{1 / 3} R_{1}^{-7(1-\varepsilon)},
$$

where we have used $R_{1} \leqq D_{1}(\delta)<1$ and $\varepsilon=\frac{1}{70} \leqq \frac{1}{21}$.

From (11) and Theorem B.3 we can find $\tilde{\alpha}(\delta)>0$ such that for $\widetilde{R} \geqq \frac{1}{2} \tilde{\alpha}(\delta) \widetilde{R}_{1}$

$$
\left(324 \pi^{2}-\frac{\delta}{2}\right) \tilde{R}^{-3} \leqq \int_{|x| \geqq \tilde{R}} \rho_{L}(x) d x \leqq\left(324 \pi^{2}+\frac{\delta}{2}\right) \tilde{R}^{-3},
$$

where we have used that $\rho_{L}(x)=\rho_{\mathrm{TF}}\left(x, R_{1}-2 r, \bar{v}\right)$. If we recall that $\int \rho_{L} d x=\bar{v}$ we can repeat the argument from Lemma 6 to get that for $R \geqq \tilde{\alpha}(\delta) R_{1}$

$$
\begin{aligned}
& -C_{\delta}^{\prime} D_{1}^{1 / 6} R^{1 / 2} R_{1}^{-\frac{7}{2}(1-\varepsilon)}+\left(324 \pi^{2}-\frac{3}{4} \delta\right) R^{-3} \leqq \bar{v}-\int_{|x| \leqq R} \theta_{+}(x)^{2} \rho_{\gamma}(x) d x \\
& \quad \leqq C_{\delta}^{\prime} D_{1}^{1 / 6} R^{1 / 2} R_{1}^{-\frac{7}{2}(1-\varepsilon)}+\left(324 \pi^{2}+\frac{3}{4} \delta\right) R^{-3} .
\end{aligned}
$$

Notice that $\bar{v}-\int_{|x| \leqq R} \theta_{+}(x)^{2} \rho_{\gamma}(x) d x=v(R)$. If $\tilde{\alpha}(\delta) R_{1} \leqq R \leqq \beta_{1} R_{1}^{(1-\varepsilon)}$ for any $\beta_{1}>0$ we obtain

$$
\begin{aligned}
& \left(-C_{\delta}^{\prime} D_{1}^{1 / 6} \beta_{1}^{-\frac{7}{2}}-\frac{3}{4} \delta+324 \pi^{2}\right) R^{-3} \leqq v(R) \leqq\left(C_{\delta}^{\prime} D_{1}^{1 / 6} \beta_{1}^{-\frac{7}{2}}+\frac{3}{4} \delta\right. \\
& \left.+324 \pi^{2}\right) R^{-3} .
\end{aligned}
$$

If we let $R_{1}$ run over $\left(\frac{4}{3} \alpha Z^{-\frac{1}{3}}, \frac{4}{5} \beta Z^{-\frac{1}{3}(1-\varepsilon)^{n}}\right)$ we find that (14) holds if

$$
\frac{4}{3} \alpha \tilde{\alpha}(\delta) Z^{\frac{1}{3}} \leqq R \leqq \beta_{1}\left(\frac{4}{5} \beta\right)^{1-\varepsilon} Z^{-\frac{1}{3}(1-\varepsilon)^{n+1}} .
$$

We choose $\beta_{1}$ such that $\beta_{1}\left(\frac{4}{5} \beta\right)^{1-\varepsilon} \geqq \beta$. Then we choose $D_{1}(\delta)$ such that $C_{\delta}^{\prime} D_{1}^{1 / 6} \beta_{1}^{-\frac{7}{2}}<\frac{1}{4} \delta$. 
Furthermore we want to make sure that the interval (15) overlaps the old interval (6), i.e.

$$
\frac{4}{3} \alpha \tilde{\alpha}(\delta) Z^{-\frac{1}{3}} \leqq \beta Z^{-\frac{1}{3}(1-\varepsilon)} .
$$

But since $\alpha Z^{-\frac{1}{3}} \leqq D(\delta)$ this holds if just $D(\delta)$ is chosen small enough.

We have proved that (1.18) holds if $R$ satisfies (7). The proof that (1.19) holds is very similar, using results corresponding to (4) and (5). It is left as an exercise to the reader.

\section{Bounds on excess charge and ionization energy}

In this final section we prove Theorem 4.

First notice that from Theorem $1, Q_{c}(Z) \leqq Z$ and

$$
I(Z) \leqq-E_{\mathrm{RHF}}\left(N_{c}(Z), Z\right) \leqq \text { const. } Z^{7 / 3}
$$

The last bound follows since $E_{\mathrm{RHF}}\left(N_{c}(Z), Z\right)$ can be estimated below by the sum of the $N_{c}(Z)$ first negative eigenvalues of the hydrogen atom.

It is then clear that in proving Theorem 4 , we can assume that $Z$ is greater than any constant. We can therefore use Theorem 3.

The method we will use to prove the excess charge bound is similar to the methods used in [SSS] and [So].

The absolute minimizer can be written as

$$
\gamma=\sum_{k} \lambda_{k} \varphi_{k} \otimes \bar{\varphi}_{k}
$$

where $\left\{\varphi_{k}\right\}$ is an orthonormal family with

$$
h_{Z}^{\gamma} \varphi_{k}=\varepsilon_{k} \varphi_{k}, \quad \varepsilon_{k} \leqq 0 .
$$

Let $R$ be a fixed radius independent of $Z$, with $R<\frac{1}{4} D$. Choose $0 \leqq \chi \in C^{\infty}\left(\mathbb{R}^{3}\right)$ with $\chi(x)=1$ if $|x| \geqq 2 R$ and $\chi(x)=0$ if $|x| \leqq R$. Then

$$
\begin{aligned}
0 & \geqq \sum_{k} \varepsilon_{k} \lambda_{k}\left(\varphi_{k},|x| \chi(x) \varphi_{k}\right) \\
& =\sum_{k} \lambda_{k}\left(\varphi_{k},|x| \chi(x) h_{Z}^{\nu} \varphi_{k}\right) \\
& =\sum_{k} \lambda_{k}\left[-\left(\varphi_{k},|x| \chi(x) \Delta \varphi_{k}\right)-\int\left|\varphi_{k}(x)\right|^{2}|x| \chi(x)\left(\frac{Z}{|x|}-\rho_{\gamma} *|x|^{-1}\right) d x\right] .
\end{aligned}
$$


Notice that the first term is real so

$$
\begin{aligned}
& \left(\varphi_{k},|x| \chi(x)(-\Delta) \varphi_{k}\right)=\int \overline{\varphi_{k}(x)}|x| \chi(x)(-\Delta) \varphi_{k} d x \\
& \quad=\int\left|\nabla \varphi_{k}\right|^{2}|x| \chi(x) d x+\operatorname{Re}\left(\int \overline{\varphi_{k}(x)} \nabla \varphi_{k}(x) \cdot \nabla(|x| \chi(x)) d x\right) \\
& \quad \geqq-\frac{1}{2} \int\left|\varphi_{k}\right|^{2} \Delta(|x| \chi(x)) d x \geqq-C R^{-1} \int_{|x| \geqq R}\left|\varphi_{k}\right|^{2} d x .
\end{aligned}
$$

Using Theorem 3 we conclude

$$
\begin{aligned}
\sum_{k} \lambda_{k}\left(\varphi_{k},|x| \chi(x)(-\Delta) \varphi_{k}\right) & \geqq-C R^{-1}\left(\int_{|x| \geqq R} \rho_{\gamma}(x) d x\right) \\
& \geqq-C R^{-1}\left(\int \chi(x) \rho_{\gamma}(x) d x+C\right) .
\end{aligned}
$$

Then from (1) we find

$$
\begin{aligned}
0 \geqq & -C R^{-1}\left(\int \chi(x) \rho_{\gamma}(x) d x+C\right)-Z \int \chi(x) \rho_{\gamma}(x) d x \\
& +\int \chi(x) \rho_{\gamma}(x)|x||x-y|^{-1} \rho_{\gamma}(y) d x d y \\
= & -C R^{-1}\left(\int \chi(x) \rho_{\gamma}(x) d x+C\right)-Z \int \chi(x) \rho_{\gamma}(x) d x \\
& +\int(1-\chi(y)) \rho_{\gamma}(y)|x-y|^{-1}|x| \chi(x) \rho_{\gamma}(x) d x d y \\
& +\frac{1}{2} \int \chi(y) \rho_{\gamma}(y) \frac{|x|+|y|}{|x-y|} \chi(x) \rho_{\gamma}(x) d x d y .
\end{aligned}
$$

In the last term we have used symmetrization. Using the triangle inequality and the definition of $v(R)$ we arrive at

$$
0 \geqq-C R^{-1}\left(\int \chi(x) \rho_{\gamma}(x) d x+C\right)-v(R) \int \chi(x) \rho_{\gamma}(x) d x+\frac{1}{2}\left(\int \chi(x) \rho_{\gamma}(x) d x\right)^{2} .
$$

Since $R$ and $\nu(R)$ are bounded by constants we get

$$
\int \chi(x) \rho_{\gamma}(x) d x \leqq \text { Const. }
$$

Then

$$
\begin{aligned}
Q_{c}(Z) & =N_{c}(Z)-Z=\int \rho_{\gamma}(x) d x-Z \\
& \leqq \int_{|x| \leqq 2 R} \rho_{\gamma}(x) d x-Z+\int \chi(x) \rho_{\gamma}(x) d x \\
& =-v(2 R)+\int \chi(x) \rho_{\gamma}(x) d x \leqq \text { Const. }
\end{aligned}
$$

since $v(2 R)>0$.

To prove the bound on the ionization energy we go back to equation (3.4). From Theorem 3 we can choose $R_{1}$ and $r$ independent of $Z$ such that

$$
\int \theta_{-}^{2} \rho_{\gamma} \leqq N_{c}(Z)-1 \text {. }
$$

Then $\mathscr{E}_{\mathrm{RHF}}\left(\theta_{-} \gamma \theta_{-}\right) \geqq E_{\mathrm{RHF}}\left(N_{c}(Z)-1, Z\right)$. Hence we just have to estimate $\mathscr{E}_{\mathrm{RHF}}\left(\theta_{-} \gamma \theta_{-}\right)-E_{\mathrm{RHF}}^{\mathrm{min}}$ from above by a constant. Or, from (3.4) it is enough to show

$$
-\operatorname{Tr}\left[h_{\bar{v}}\left(\theta_{+} \gamma \theta_{+}\right)\right]+C_{\theta} r^{-2} \Delta Q\left(R_{1}, r\right)+4 R_{1}^{-2} r \Delta Q\left(R_{1}, r\right)^{2} \leqq \text { Const. }
$$


But since $\bar{v}, R_{1}, r$ are bounded by constants and

$$
\begin{aligned}
\int \theta_{+}(x)^{2} \rho_{y}(x) d x & =N_{c}(Z)-\int \theta_{-}(x)^{2} \rho_{y}(x) d x \\
& =Q_{c}(Z)+\bar{v}<\text { Const. }
\end{aligned}
$$

We immediately conclude (3).

This completes the proof of Theorem 4.

\section{Appendix A. The RHF minimization problem}

In this appendix we prove Theorem 1 . We first notice

Lemma A1. The map

$$
\text { Trace class operators in } L^{2}\left(\mathbb{R}^{3}, \mathbb{C}^{3}\right) \ni \gamma \mapsto \rho_{y} \in L^{1}\left(\mathbb{R}^{3}\right)
$$

is continuous and linear.

The proof of this is left to the reader.

For all rotations $\Omega \in S O(3)$ there corresponds a unitary operator $U_{\Omega}$ on $L^{2}\left(\mathbb{R}^{3}, \mathbb{C}^{2}\right)$. We denote $\gamma_{\Omega}=U_{\Omega}^{-1} \gamma U_{\Omega}$ for $\gamma \in G$, where the set $G$ was defined in (1.10). Then $\rho_{\gamma_{\Omega}}(x)=\rho_{\gamma}\left(\Omega^{-1} x\right)$. It is clear that $\mathscr{E}_{\mathrm{RHF}}(\gamma)=\mathscr{E}_{\mathrm{RHF}}\left(\gamma_{\Omega}\right)$.

If $d \Omega$ denotes the Haar measure on $S O(3)$ we get from the convexity of $\mathscr{E}_{\text {RHF }}$

$$
\mathscr{E}_{\mathrm{RHF}}\left(\int \gamma_{\Omega} d \Omega\right) \leqq \int \mathscr{E}_{\mathrm{RHF}}\left(\gamma_{\Omega}\right) \mathrm{d} \Omega=\mathscr{E}_{\mathrm{RHF}}(\gamma)
$$

Given $N>0$, it is then clear from the above spherical averaging procedure that we can choose a sequence $\left\{\gamma^{(n)}\right\}$ in $G$ with $\operatorname{Tr} \gamma^{(n)} \leqq N$ and $\gamma_{\Omega}^{(n)}=\gamma^{(n)}$ for all $\Omega \in S O(3)$, such that

$$
\lim _{n \rightarrow \infty} \mathscr{E}_{\mathrm{RHF}}\left(\gamma^{(n)}\right)=E_{\mathrm{RHF}}(N, Z)
$$

Let $\rho^{(n)}=\rho_{\gamma^{(n)}}$. Then $\rho^{(n)}$ is spherically symmetric.

Since $\gamma^{(n)}$ is minimizing $\operatorname{Tr}\left[h_{Z} \gamma^{(n)}\right]$ is a bounded sequence. It then follows from Kato's inequality

$$
\operatorname{Tr}\left[\gamma^{(n)} V\right] \leqq \varepsilon \operatorname{Tr}\left[-\Delta \gamma^{(n)}\right]+C_{\varepsilon} \operatorname{Tr} \gamma^{(n)}, \quad \text { all } \varepsilon>0
$$

with $V=Z /|x|$ acting as a multiplication operator, that $\operatorname{Tr}\left[-\Delta \gamma^{(n)}\right]$ is bounded.

$\tilde{\gamma}^{(n)}=(1-\Delta)^{1 / 2} \gamma^{(n)}(1-\Delta)^{1 / 2}$ defines a sequence of positive trace class operators on $L^{2}\left(\mathbb{R}^{3}, \mathbb{C}^{2}\right)$ with bounded trace norms

$$
\operatorname{Tr}\left[\gamma^{(n)}\right]=\operatorname{Tr}\left[(1-\Delta) \gamma^{(n)}\right] .
$$

Especially $\left\{\tilde{\gamma}^{(n)}\right\}$ is a sequence of Hilbert-Schmidt operators with bounded Hilbert-Schmidt norm.

Since the space $\mathscr{C}^{2}\left(L^{2}\left(\mathbb{R}^{3}, \mathbb{C}^{2}\right)\right)$ of Hilbert-Schmidt operators on $L^{2}\left(\mathbb{R}^{3}, \mathbb{C}^{2}\right)$ is a Hilbert space we can assume by going to a subsequence that $\tilde{\gamma}^{(n)}$ converges 
weakly in $\mathscr{C}^{2}\left(L^{2}\left(\mathbb{R}^{3}, \mathbb{C}^{2}\right)\right.$ ). Thus there is a $\tilde{\gamma}^{(\infty)} \in \mathscr{C}^{2}$ such that for all $W \in \mathscr{C}^{2}$, $\operatorname{Tr}\left[W \tilde{\gamma}^{(n)}\right] \rightarrow \operatorname{Tr}\left[W \tilde{\gamma}^{(\infty)}\right]$. Let

$$
\gamma^{(\infty)}=(1-\Delta)^{-\frac{1}{2}} \tilde{\gamma}^{(\infty)}(1-\Delta)^{-\frac{1}{2}}
$$

Choose an orthonormal basis $\left\{\psi_{k}\right\}$ in $L^{2}\left(\mathbb{R}^{3}, \mathbb{C}^{2}\right)$ such that each $\psi_{k} \in H^{1}\left(\mathbb{R}^{3}, \mathbb{C}^{2}\right)$. From the weak convergence in $\mathscr{C}^{2}$ it follows that

$$
\lim _{n \rightarrow \infty}\left(\psi_{k}, \gamma^{(n)} \psi_{k}\right)=\lim _{n \rightarrow \infty}\left((1-\Delta)^{-\frac{1}{2}} \psi_{k}, \tilde{\gamma}^{(n)}(1-\Delta)^{-\frac{1}{2}} \psi_{k}\right)=\left(\psi_{k}, \gamma^{(\infty)} \psi_{k}\right)
$$

Here ( , ) denotes the inner product in $L^{2}\left(\mathbb{R}^{3}, \mathbb{C}^{2}\right)$. Likewise

$$
\lim _{n \rightarrow \infty}\left(\psi_{k},(-\Delta)^{1 / 2} \gamma^{(n)}(-\Delta)^{1 / 2} \psi_{k}\right)=\left(\psi_{k},(-\Delta)^{1 / 2} \gamma^{(\infty)}(-\Delta)^{1 / 2} \psi_{k}\right) .
$$

Since $\gamma^{(n)} \geqq 0$ and $(-\Delta)^{1 / 2} \gamma^{(n)}(-\Delta)^{1 / 2} \geqq 0$ it follows from Fatou's lemma for sequences that

$$
\operatorname{Tr} \gamma^{(\infty)}=\sum_{k}\left(\psi_{k}, \gamma^{(\infty)} \psi_{k}\right) \leqq \underline{\lim } \operatorname{Tr} \gamma^{(n)} \leqq N
$$

and

$$
\operatorname{Tr}\left(-\Delta \gamma^{(0)}\right) \leqq \underline{\lim } \operatorname{Tr}\left(-\Delta \gamma^{(n)}\right)
$$

We clearly also have $0 \leqq \gamma^{(\infty)} \leqq 1$.

Since $\gamma^{(\infty)}$ is spherically symmetric so is $\rho^{(\infty)}(x)$. Hence $\rho^{(\infty)} *|x|^{-1}$ $\leqq|x|^{-1} \int \rho^{(\infty)}(x) d x \leqq N|x|^{-1}$. We then get from Kato's inequality again that

$$
D\left(\rho^{(\infty)}, \rho^{(\infty)}\right)=\operatorname{Tr}\left[\rho^{(\infty)} *|x|^{-1} \cdot \gamma^{(\infty)}\right]<\infty .
$$

For any compactly supported function $f$ with $0 \leqq f(x) \leqq C / x \mid$ the operator $(1-\Delta)^{-\frac{1}{2}} \cdot f \cdot(1-\Delta)^{-\frac{1}{2}}$ is Hilbert Schmidt which can easily be seen from the integral kernel representation of $(1-\Delta)^{-1}$.

From this we can now prove that

$$
\lim _{n \rightarrow \infty} \int \rho^{n}(x) \frac{Z}{|x|} d x=\int \rho^{(\infty)}(x) \frac{Z}{|x|} d x .
$$

Write $Z|x|^{-1} \equiv V(x)=V \cdot \chi_{r}+V\left(1-\chi_{r}\right)$, where $\chi_{r}$ is the characteristic function for the set $\{|x| \leqq r\}$. Now

$$
V\left(1-\chi_{r}\right)(x)=Z_{r} *|x|^{-1} \quad \text { for }|x| \geqq r,
$$


where $Z_{r}$ is the uniform charge distribution over $\{|x| \leqq r\}$ with total charge $Z$. Thus

$$
\begin{aligned}
& \left|\int\left(\rho^{(n)}-\rho^{(\infty)}\right) V\left(1-\chi_{r}\right) d x\right| \leqq 2 D\left(\left|\rho^{(n)}-\rho^{(\infty)}\right|, Z_{r}\right) \\
& \quad \leqq 2 D\left(\rho^{(n)}+\rho^{(\infty)}, \rho^{(n)}+\rho^{(\infty)}\right)^{1 / 2} \cdot D\left(Z_{r}, Z_{r}\right)^{1 / 2} \\
& \quad \leqq 2\left(D\left(\rho^{(n)}, \rho^{(n)}\right)^{1 / 2}+\left(D\left(\rho^{(\infty)}, \rho^{(\infty)}\right)^{1 / 2}\right) \cdot D\left(Z_{r}, Z_{r}\right)^{1 / 2}\right. \\
& \quad \leqq \text { Const. } D\left(Z_{r}, Z_{r}\right)^{1 / 2} \underset{r \rightarrow \infty}{\longrightarrow} 0 .
\end{aligned}
$$

We have used (7) and that since $\gamma^{(n)}$ is minimizing $D\left(\rho^{(n)}, \rho^{(n)}\right)$ is bounded.

On the other hand

$$
\int \rho^{(n)}(x) V(x) \chi_{r}(x) d x=\frac{\operatorname{Tr}\left[\gamma^{(n)} V \chi_{r}\right]=\operatorname{Tr}\left[\hat{\gamma}^{(n)}(1-\Delta)^{-\frac{1}{2}} \cdot V \chi_{r} \cdot(1-\Delta)^{-\frac{1}{2}}\right]}{\underset{n \rightarrow \infty}{\longrightarrow} \operatorname{Tr}\left[\gamma^{(\infty)}(1-\Delta)^{-\frac{1}{2}} \cdot V \chi_{r} \cdot(1-\Delta)^{-\frac{1}{2}}\right] .}
$$

Since $(1-\Delta)^{-\frac{1}{2}} \cdot V \chi_{r} \cdot(1-\Delta)^{-\frac{1}{2}}$ is Hilbert-Schmidt. This gives (8).

We likewise conclude that $\lim _{n \rightarrow \infty} D\left(\rho^{(n)}, \rho^{(\infty)}\right)=D\left(\rho^{(\infty)}, \rho^{(\infty)}\right)$. But

$$
D\left(\rho^{(n)}, \rho^{(\infty)}\right) \leqq D\left(\rho^{(n)}, \rho^{(n)}\right)^{1 / 2} D\left(\rho^{(\infty)}, \rho^{(\infty)}\right)^{1 / 2}
$$

and we conclude that

$$
D\left(\rho^{(\infty)}, \rho^{(\infty)}\right) \leqq \underline{\lim } D\left(\rho^{(n)}, \rho^{(n)}\right) .
$$

From (5)-(9) we see that $\mathscr{E}_{\mathrm{RHF}}\left(\gamma^{(\infty)}\right)=E(N, Z)$.

Recalling that $\mathscr{E}_{\mathrm{RHF}}$ is a convex functional because $\rho \mapsto D(\rho, \rho)$ is strictly convex we conclude (a)-(c) of Theorem 1 except for the bound $Z \leqq N_{c}(Z) \leqq 2 Z$.

We now prove Theorem 1 (d). We can write $\gamma^{(\infty)}=\gamma=\sum_{k=1}^{\infty} \lambda_{k} \varphi_{k} \otimes \bar{\varphi}_{k}$ with 1 $\geqq \lambda_{1} \geqq \ldots \geqq 0$. Choose $K \geqq 0$ such that $K+1$ is the smallest number with $\lambda_{K+1}<1$. If $u \perp \operatorname{span}\left\{\varphi_{1}, \ldots, \varphi_{K}\right\}$ with $\|u\|=1$, we define for $j \in\{1, \ldots, K\}$

$$
\gamma_{\varepsilon}^{(j)}=\sum_{k \neq j} \lambda_{k} \varphi_{k} \otimes \bar{\varphi}_{k}+\frac{1}{1+m \varepsilon^{2}}\left(\varphi_{j}+\varepsilon u\right) \otimes\left(\bar{\varphi}_{j}+\varepsilon \bar{u}\right)
$$

It is easy to see that we can choose $m>1$ such that for $\varepsilon$ small enough, $0 \leqq \gamma_{\varepsilon}^{(j)} \leqq 1$ and $\operatorname{Tr}\left[\gamma_{\varepsilon}^{(j)}\right] \leqq N$. Then

$$
0=\left.\frac{d}{d \varepsilon} \mathscr{E}_{\mathrm{RHF}}\left(\gamma_{\varepsilon}^{(j)}\right)\right|_{\varepsilon=0}=\left(\varphi_{j}, h_{Z}^{\gamma} u\right)+\left(u, h_{Z}^{\gamma} \varphi_{j}\right)
$$

Thus $\left(u, h_{Z}^{y} \varphi_{j}\right)=0$. This shows that $h_{Z}^{y}$ maps $\operatorname{span}\left\{\varphi_{1}, \ldots, \varphi_{K}\right\}$ into itself. This space is therefore a sum of eigenspaces for $h_{Z}^{\gamma}$.

We can rewrite

$$
\mathscr{E}_{\mathbf{R H F}}(\gamma)=\operatorname{Tr}\left[h_{Z}^{\gamma} \gamma\right]-D\left(\rho_{\gamma}, \rho_{\gamma}\right)
$$


The first term is smallest if $\varphi_{1}, \ldots, \varphi_{K}$ are eigenvectors for $h_{z}^{\gamma}$. On the other hand $D\left(\rho_{\gamma}, \rho_{\gamma}\right)$ is independent of the basis for $\operatorname{span}\left\{\varphi_{1}, \ldots, \varphi_{K}\right\}$. Hence we conclude that $\varphi_{1}, \ldots, \varphi_{K}$ are eigenvectors. That $\varphi_{K+1}, \varphi_{K+2}, \ldots$, are eigenvectors is proved by showing in the same manner that $h_{z}^{\gamma}$ leaves $\operatorname{span}\left\{\varphi_{1}, \ldots, \varphi_{K}, \varphi_{j}\right\}$ invariant for $j=K+1, \ldots$.

We are left with proving $Z \leqq N_{c}(Z) \leqq 2 Z$. The upper bound follows as in [L3]. If $N<Z$ it is clear that $h_{Z}^{\gamma}$ has infinitely many eigenvalues. We define $K$ as before. Then $K \leqq N$, and we can choose $u \perp \operatorname{span}\left\{\varphi_{1}, \ldots, \varphi_{K}\right\}$ such that $u$ is a normalized eigenfunction of $h_{Z}^{\nu}$. Then for $\varepsilon>0$ small enough

$$
\gamma_{\varepsilon}=\gamma+\varepsilon \boldsymbol{u} \otimes \bar{u}
$$

satisfies $0 \leqq \gamma_{\varepsilon} \leqq 1$ and $\operatorname{Tr} \gamma_{\varepsilon}=N+\varepsilon$. Furthermore

$$
\mathscr{E}_{\mathrm{RHF}}\left(\gamma_{\varepsilon}\right)=\mathscr{E}_{\mathrm{RHF}}(\gamma)+\varepsilon\left(u, h_{Z}^{\gamma} u\right)+\varepsilon^{2} D\left(|u|^{2},|u|^{2}\right) .
$$

It thus follows that $E(N+\varepsilon, Z)<E(N, Z)$ and hence $N_{c}(Z) \geqq Z$.

(1.13) holds because it is true for Hartree-Fock theory and it is easy to see that the exchange term is bounded by $C \cdot Z^{5 / 3}$.

\section{Appendix B. Exterior TF models}

The Thomas-Fermi theory of an atom with nuclear charge $\bar{v}$ is defined from the functional

$$
\mathscr{E}_{T F}(\rho)=\frac{3}{5}\left(3 \pi^{2}\right)^{2 / 3} \int \rho(x)^{5 / 3} d x-\bar{v} \int|x|^{-1} \rho(x) d x+D(\rho, \rho) .
$$

Usually this functional is defined on densities $\rho$ on all of $\mathbb{R}^{3}$. Here we will restrict the functional to densities supported outside some ball.

We define the exterior TF energy corresponding to a radius $\bar{R}>0$

$$
\begin{array}{r}
E_{\mathbf{T F}}^{\operatorname{ex}}(\lambda, \bar{R}, \bar{v})=\inf \left\{\mathscr{O}_{T F}(\rho) \mid \rho \in L^{5 / 3}\left(\mathbb{R}^{3}\right) \cap L^{1}\left(\mathbb{R}^{3}\right),\right. \\
\left.\operatorname{supp} \rho \subseteq\{|x| \geqq \bar{R}\}, \int \rho(x) d x \leqq \lambda\right\} .
\end{array}
$$

It is clear that $E_{\mathrm{TF}}^{\mathrm{ex}} \geqq E_{\mathrm{TF}}$, where $E_{\mathrm{TF}}$ is the usual TF energy, i.e., corresponding to $\bar{R}=0$.

As for regular TF theory (see [LS1] or [L4]) we can prove that there exists $\lambda_{c}, 0<\lambda_{c} \leqq \infty$ such that the exterior problem has a unique minimizer $\rho$ with $\int \rho(x) d x=\lambda$ if $\lambda \leqq \lambda_{c}$. We will prove below in Lemma B 1 that $\lambda_{c}<\infty$.

For $\lambda \leqq \lambda_{c}$ the minimizer $\rho(x)$ satisfies

$$
\left(3 \pi^{2} \rho(x)\right)^{2 / 3}=\left[\varphi_{\rho}(x)-\mu\right]_{+} \quad \text { on }|x| \geqq \widetilde{R}
$$

for a Lagrange multiplier $\mu \geqq 0$. Here $[t]_{+}=t$ if $t \geqq 0,[t]_{+}=0$ if $t<0$, and

$$
\varphi_{\rho}(x)=\frac{\bar{v}}{|x|}-\rho *|x|^{-1} \text {. }
$$

Lemma B1. $\lambda_{\mathrm{c}}=\bar{v}$. 
Proof. Let $S=\left\{x\left|\varphi_{\rho}(x)<0,\right| x \mid \geqq \bar{R}\right\}$. We want to prove that $S=\emptyset$. Since $\varphi$ is radial and subharmonic it follows that if $\varphi<0$ on $\{|x|=\bar{R}\}$ then $\varphi<0$ on $\{|x| \geqq \bar{R}\}$. From (3) we get that $\rho=0$. Thus $\varphi \geqq 0$ on $\{|x|=\bar{R}\}$ and if follows that $S$ is open. $\varphi$ is harmonic on $S$ and is 0 on the boundary, hence $\varphi=0$ on $S$ which is a contradiction unless $S=\emptyset$.

We have proved that $\varphi_{\rho} \geqq 0$ for any minimizer. Hence $\lambda=\int \rho(x) d x \leqq \bar{v}$, i.e., $\lambda_{c} \leqq \bar{v}$.

Proving $\lambda \geqq \bar{v}$ follows as in [L4] Theorem 3.18.

When $\lambda=\bar{v}$ we get the absolute minimizer corresponding to $\mu=0$. This is the case that will interest us from now on, we denote it

$$
\rho(x)=\rho_{\mathrm{TF}}(x, \bar{R}, \bar{v}) \quad \varphi(x)=\varphi_{\mathrm{TF}}(x, \bar{R}, \bar{v}) .
$$

$\varphi$ is the unique solution to

$$
\left\{\begin{array}{l}
\frac{3}{4} \pi \Delta \varphi=\varphi^{3 / 2}, \quad|x|>\bar{R} \\
\left.\partial_{r} \varphi\right|_{|x|=\bar{R}}=-\bar{v} \bar{R}^{-2}
\end{array}\right.
$$

$\partial_{r}$ denotes the radial derivative. The boundary condition follows from the spherical symmetry since

$$
\partial_{r} \varphi(x)=|x|^{-2}\left(\int_{|y| \leqq|x|} \rho(y) d y-\bar{v}\right) .
$$

$\varphi$ has the scaling property

$$
\varphi(x, \bar{R}, \bar{v})=\bar{R}^{-4} \varphi\left(\bar{R}^{-1} x, 1, \bar{R}^{3} \bar{v}\right) .
$$

Lemma B2. If $\bar{v}_{1}<\bar{v}_{2}$ then $\varphi\left(x, \bar{R}, \bar{v}_{1}\right)<\varphi\left(x, \bar{R}, \bar{v}_{2}\right)$.

Proof. We cannot have $\varphi_{1}(x)=\varphi_{1}\left(x, \bar{R}, \bar{v}_{2}\right)>\varphi\left(x, \bar{R}, \bar{v}_{1}\right)=\varphi_{2}(x)$ for all $|x| \geqq \bar{R}$. Since this would imply from (3) that $\bar{v}_{1}=\int \rho_{1} d x>\int \rho_{2} d x=\bar{v}_{2}$.

On the other hand $\varphi_{1}$ and $\varphi_{2}$ cannot intersect. Indeed if $|x|=r_{1}$ is an intersection, then there will be another intersection $r_{2}>r_{1}$ (possible $r_{2}=\infty$ ) such that in $\left\{r_{1}<|x|<r_{2}\right\} \varphi^{\prime}(x)<\varphi^{\prime \prime}(x)$ where $\varphi^{\prime}, \varphi^{\prime \prime}$ represent $\varphi_{1}, \varphi_{2}$ in some order. Then $\Delta\left(\varphi^{\prime}-\varphi^{\prime \prime}\right)=\operatorname{const}\left(\varphi^{\prime 3 / 2}-\varphi^{\prime \prime 3 / 2}\right)<0$ but this implies $\varphi^{\prime}>\varphi^{\prime \prime}$ on $\left\{r_{1}<|x|\right.$ $\left.<r_{2}\right\}$.

If $\bar{v} \cdot \bar{R}^{3} \geqq \kappa>0$ we find from (8)

$$
\varphi(x, \bar{R}, \bar{v})=\bar{R}^{-4} \varphi\left(\bar{R}^{-1} x, 1, \bar{v} \bar{R}^{3}\right) \geqq \bar{R}^{-4} \varphi\left(\bar{R}^{-1} x, 1, \kappa\right) .
$$

It is easy to prove, see [L4] Theorem 2.10 or [LS1] Sect. V.2 that

$$
\lim _{|y| \rightarrow \infty}|y|^{4} \varphi(y, 1, \kappa)=81 \pi^{2} .
$$


From [So] Lemma 11 we get for $|x|>$ Const $\bar{R}$

$$
\varphi(x, \bar{R}, \bar{v}) \leqq\left(81 \pi^{2}+C(|x| / \bar{R})^{4-\tau}\right)|x|^{-4},
$$

where $\tau=\frac{1}{2}+\frac{\sqrt{73}}{2}>4$.

Putting together (9)-(11) we have proved

Theorem B3. If $\bar{v} \cdot \vec{R}^{3} \geqq \kappa>0$ and $\delta_{1}>0$ we can find $\alpha\left(\kappa, \delta_{1}\right)>0$ such that for $R=|x| \geqq \alpha\left(\kappa, \delta_{1}\right) \bar{R}$

$$
\begin{aligned}
& \left(81 \pi^{2}-\delta_{1}\right)|x|^{-4} \leqq \varphi_{\mathrm{TF}}(x, \bar{R}, \bar{v}) \leqq\left(81 \pi^{2}+\delta_{1}\right)|x|^{-4} \\
& \left(324 \pi^{2}-\delta_{1}\right) R^{-3} \leqq \int_{|x| \geqq R} \rho_{\mathrm{TF}}(x, \bar{R}, \bar{v}) d x \leqq\left(324 \pi^{2}+\delta_{1}\right) R^{-3} .
\end{aligned}
$$

\section{References}

[BL] Benguria, R., Lieb, E.H.: The most negative ion in the Thomas-Fermi-von Weizsäcker theory of atoms and molecules. J. Phys. B18, 1045-1059 (1985)

[FS1] Fefferman, C.L., Seco, L.: Asymptotic neutrality of large ions. Comm. Math. Phys. 128, 109-130(1990)

[FS2] Fefferman, C.L., Seco, L.: On the energy of a large atom. Bull. Am. Math. Soc., 23, 525-529 (1990)

[H] Hartree, D.: The wave mechanics of an atom with a non-Coulomb central field. Part I. Theory and methods. Proc. Comb. Phil. Soc. 24, 89-132 (1928)

[L 1] Lieb, E.H.: A lower bound for Coulomb energies. Phys. Lett. A 70, 444446 (1979)

[L2] Lieb, E.H.: A variational principle for many-fermion systems. Phys. Rev. Lett. 46, $457-459(1981)$

[L3] Lieb, E.H.: Bound on the maximum negative ionization of atoms and molecules. Phys. Rev. A29, 3018-3028 (1984)

[L4] Lieb, E.H.: Thomas-Fermi and related theories of atoms and molecules. Rev. Mod. Phys. 53, 603-641 (1981)

[L5] Lieb, E.H.: The number of bound states of one-body Schrödinger operators and the Weyl problem. AMS Proc. Symp. Pure Math. 36, 241-252 (1980)

[LS1] Lieb, E.H., Simon, B.: The Thomas-Fermi theory of atoms, molecules, and solids. Adv. Math. 23, 22-116 (1977)

[LS2] Lieb, E.H., Simon, B.: The Hartree-Fock Theoty for Coulomb Systems. Comm. Math. Phys. 53, 185-194 (1977)

[LSST] Lieb, E.H., Sigal, I.M., Simon, B., Thirring, W.: Asymptotic neutrality of large Zatoms. Commun. Math. Phys. 116, 635-644 (1988)

[R 1] Ruskai, M.B.: Absence of discrete spectrum in highly negative ions. Commun. Math. Phys. 82, 457-469 (1982)

[R2] Ruskai, M.B.: Absence of discrete spectrum in highly negative ions. II. Commun. Math. Phys. 85, 325-327 (1982)

[SSS] Seco, L., Sigal, I.M., Solovej, J.P.: Bound on the ionization energy of large atoms. Commun. Math. Phys. 131, 307-315 (1990)

[SW] Siedentop, H., Weikand, R.: On Some Basic Properties of Density Functionals for Angular Momentum Channels. Rep. Math. Phys. 24, 193-218 (1986)

[Si 1] Sigal, I.M.: In: Proc. of the VI International Congress on Mathematical Physics., Berlin, 1981

[Si2] Sigal, I.M.: Geometric methods in the quantum many-body problem. Nonexistence of very negative ions. Commun. Math. Phys. 85, 309-324 (1982)

[Si3] Sigal, I.M.: How many electrons can a nucleus bind? Ann. Phys. 157, 307-320 (1984)

[So] Solovej, J.P.: Universality in the Thomas-Fermi-von Weizsäcker model of atoms and molecules. Commun. Math. Phys. 129, 561-598 (1990)

[T] Thirring, W.: A lower bound with the best possible constant for Coulomb Hamiltonians. Commun. Math. Phys. 79, 1-7 (1981) 